\title{
Elementos de la cultura cabécar relacionados con nociones matemáticas que se estudian a nivel de educación primaria en Costa Rica
}

Alejandra Sánchez Ávila

Encargada, Cátedra Didáctica de la Matemática, Escuela de Ciencias de la Educación UNED, Costa Rica

alsanchez@uned.ac.cr

Recibido: 11 de agosto del 2016

Corregido: 26 de setiembre del 2016

Aceptado: 23 de marzo del 2017

\section{Resumen}

El propósito de este artículo es mostrar elementos de la cultura cabécar relacionados con nociones matemáticas que se incluyen en el Programa de Estudios de l y ll ciclos de la Educación General Básica costarricense, a partir de la investigación titulada "Educación para todos: La cultura cabécar y la matemática en I y ll ciclos de la Enseñanza General Básica en Costa Rica". Los datos se obtienen mediante la elaboración de grupos focales y con la participación de los estudiantes de la Carrera Bachillerato en Ciencias de la Educación en I y ll ciclos con énfasis en Lengua y cultura cabécar y a través de entrevistas a personal docente de tres escuelas en comunidades indígenas. Entre los hallazgos más importantes están la construcción de la base del rancho cultural sin la utilización de los instrumentos occidentales tradicionales, así como las formas de medición y conteo de diferentes objetos. Una de las implicaciones más relevantes es la necesidad de contextualizar el Programa de Estudio de matemática para las poblaciones indígenas.

Palabras clave: Educación indígena, interculturalidad, enseñanza primaria, enseñanza de la matemática, cultura indígena, Etnomatemática.

\section{Abstract \\ Elements from cabécar culture related to mathematical notions currently studied in Costa Rican Elementary School}

The purpose of this article is to show some elements from cabécar culture that are related to mathematical notions currently included in the official Mathematica Education Study Program of I and II cycle of Costa Rican Elementary School, from the research entitled "Education for everyone: cabécar culture and mathematics in I and II cycle of Elementary Education in Costa Rica". The data is obtained through the technique of focal groups, and along with the participation of under degree and degree students from Education Sciences for I and II cycle with emphasis in cabécar Culture and Language career, and through personal interviews to actual teachers from three indigenous communities. Among the important discoveries are the construction of a cultural shack without using traditional occidental instruments, and the ways of counting and measuring different objects. One of the most important implications is the need of contextualizing the official Mathematical Education Study Program for aboriginal populations.

Key words: Indigenous education, interculturalism, primary education, teaching mathematics, indigenous culture, Ethnomathematics. 


\section{INTRODUCCIÓN}

En Costa Rica existe un programa de estudios único, por asignatura, para toda la población nacional, por lo que, el estudiantado de los territorios indígenas debe acoplarse también a los contenidos establecidos. Por tanto, son afectados directamente con una educación asignada y como señalan Bozzoli y Guevara (2002) “(...) ha sido históricamente, un factor de destrucción cultural en los pueblos indígenas. La educación convencional, impuesta por el Estado en forma sistemática desde los años cuarenta, ha provocado la pérdida de las lenguas vernáculas, la desvalorización de la cultura y la pérdida consecuente de tradiciones culturales" (pp. 38-39).

Las Naciones Unidas (2008) se manifiestan en relación con los derechos de los pueblos indígenas, en los siguientes artículos:

Artículo 14

1. Los pueblos indígenas tienen derecho a establecer y controlar sus sistemas e instituciones docentes que impartan educación en sus propios idiomas, en consonancia con sus métodos culturales de enseñanza y aprendizaje.

2. Los indígenas, en particular los niños, tienen derecho a todos los niveles y formas de educación del Estado sin discriminación.

3. Los Estados adoptarán medidas eficaces, conjuntamente con los pueblos indígenas, para que las personas indígenas, en particular los niños, incluidos los que viven fuera de sus comunidades, tengan acceso, cuando sea posible, a la educación en su propia cultura y en su propio idioma. (p.7)

Artículo 15

1. Los pueblos indígenas tienen derecho a que la dignidad y diversidad de sus culturas, tradiciones, historias y aspiraciones queden debidamente reflejadas en la educación y la información pública.

2. Los Estados adoptarán medidas eficaces, en consulta y cooperación con los pueblos indígenas interesados, para combatir los prejuicios y eliminar la discriminación y promover la tolerancia, la comprensión y las buenas relaciones entre los pueblos indígenas y todos los demás sectores de la sociedad. (pp. 7-8)

La realidad de la educación indígena costarricense está alejada de cumplir con estos derechos en su totalidad, por lo que los resultados expuestos en este artículo pretenden hacer un pequeño aporte a la contextualización de algunos contenidos matemáticos que se estudian obligatoriamente a nivel de Primer ciclo de la Educación General Básica, de tal manera que en su desarrollo se puedan relacionar con elementos propios de la cultura, en este caso, cabécar.

La información puede ser útil a docentes que imparten matemática en territorios indígenas cabécares, a productores de material didáctico, a estudiantes de primaria, entre otros.

En Costa Rica existen veintidós territorios indígenas, en los cuales habitan ocho etnias, entre ellas la cabécar, que es a la que se refiere el artículo. En la investigación que sirve de fundamento, se trabajó específicamente en la zona de Turrialba, en Chirripó, con maestros indígenas y no indígenas de asignaturas básicas, maestros indígenas de Lengua y Cultura cabécar, estudiantes universitarios de la carrera Bachillerato en Ciencias de la Educación en I y II ciclos con énfasis en lengua y cultura cabécar. 
El proyecto se realizó durante el 2010, 2011 e inicios del 2012. Las actividades se llevaron a cabo en tres escuelas de Chirripó: Villa Damaris, Alto Quetzal y Jaktain, (de los circuitos 06 y 07 de la Región Educativa de Turrialba) y en la Sede del Atlántico de la Universidad de Costa Rica.

\section{Objetivos}

Existen dos objetivos específicos que se logran con este artículo:

- Investigar en la cultura cabécar la existencia de nociones matemáticas como: medición, orden, tiempo, cantidad, tamaño, espacio y operaciones fundamentales.

- Relacionar los elementos de la cosmovisión cabécar (rituales, cotidianeidad, organización social, relaciones interpersonales y con el entorno, actividades productivas y reproductivas) con las actividades matemáticas universales según Bishop y con los contenidos matemáticos de I y II Ciclos de la Enseñanza General Básica.

\section{Marco referencial}

Tomando en consideración al estudiante como una persona que se desenvuelve en diferentes ámbitos: familia, escuela y en cualquier espacio del entorno en el que se desarrolla, en forma individual o grupal, se percibe la necesidad de propuestas educativas diversas, que tomen en cuenta la cultura y tradiciones de las poblaciones objeto, por tanto, se considera la educación indígena como aquel sistema donde los niños y las niñas de los territorios indígenas tengan derecho a aprender a leer y escribir en su idioma materno, además que en los correspondientes programas educativos integren conocimientos propios de su cultura, cosmovisión, organización política, económica y social. Dentro del proceso educativo se debe buscar el dominio del español, como idioma oficial del país, de forma tal que cualquier propuesta curricular permita el desarrollo de programas educativos bilingües.

Según Olivé (2004) el término multiculturalidad se entiende como:

... una realidad factual—, se refiere a la presencia de minorías étnicas conformadas por efecto de las migraciones internacionales, tales como los árabes, africanos y latinos que habitan en algunos países europeos como Francia, Alemania, España; así como los chinos, cubanos, mexicanos, puertorriqueños y demás que habitan en Estados Unidos; minorías nacionales —los ya mencionados casos de España, Canadá y Rusia-; o bien, de pueblos indígenas, que coexisten con sectores dominantes de las sociedades de las que forman parte en condiciones de subalternidad y subordinación. También se utiliza a menudo el término de pluriculturalidad, como sinónimo del de multiculturalidad, y ambos se refieren a "Las situaciones de hecho en las que coexisten pueblos y culturas diversos. Bajo esta acepción, se trata de términos factuales. Podemos decir igualmente que son términos descriptivos, porque describen un aspecto de la realidad social de nuestro país, de otros países y del mundo" (p. 22).

Similarmente, Schmelkes (2006) se refiere a la interculturalidad en los siguientes términos:

No se trata de un concepto descriptivo, sino de una aspiración. Se refiere precisamente a la relación entre las culturas y califica esta relación. La interculturalidad supone que entre los grupos culturales distintos existen relaciones basadas en el respeto y desde planos de igualdad. La interculturalidad 
no admite asimetrías, es decir, desigualdades entre culturas mediadas por el poder, que benefician a un grupo cultural por encima de otro u otros. Como aspiración, la interculturalidad forma parte de un proyecto de nación (p. 2).

En la misma línea de la multiculturalidad e interculturalidad en los últimos años, matemáticos como D'Ambrosio (2008) plantearon la necesidad de desarrollar estrategias metodológicas y programas curriculares que consideren a las minorías culturales, las cuales no han sido tomadas en cuenta por los organismos políticos y técnicos encargados de desarrollar las políticas educativas para todo el territorio nacional. Según los autores, existe una matemática con características propias e inherentes a la cultura de cada pueblo denominada Etnomatemáticas. D'Ambrosio (2008) la refiere como "la matemática practicada por grupos culturales, tales como comunidades urbanas y rurales, grupos de trabajadores, grupos de profesionales, niños de cierta edad, sociedades indígenas y otros que se identifican por objetivos o tradiciones comunes" (p. 9).

Al respecto Bishop (2000) indica que "se refiere tanto al estudio de las relaciones entre las matemáticas y la cultura como a las prácticas matemáticas concretas que se llevan a cabo dentro de las comunidades donde se halla ubicada la escuela" (p. 40).

Bishop (1999) propone la existencia de seis actividades matemáticas universales, por cuanto están presentes en todas las culturas del mundo. Al respecto, Oliveras y Gavarrete (2012) las resumen en el siguiente cuadro:

TABLA 1

Actividades matemáticas según Bishop

Actividad

CONTAR

$\begin{array}{ll}\text { LOCALIZAR } & \begin{array}{l}\text { Relacionada con dimensiones, coordenadas, ejes, caminos, redes, simetría, topología, } \\ \text { distancias, direcciones y lugares geométricos }\end{array} \\ \text { MEDIR } & \begin{array}{l}\text { Relacionada con el orden, el tamaño, las unidades, los sistemas de medida, precisión y la } \\ \text { magnitud continua. }\end{array} \\ \text { DISENAR } & \begin{array}{l}\text { Relacionada con la forma, la regularidad, las pautas, las construcciones, los dibujos y la } \\ \text { representación geométrica. }\end{array} \\ \text { JUGAR } & \begin{array}{l}\text { Relacionada con las reglas de los juegos, los procedimientos, los planes, los modelos de } \\ \text { juego, la satisfacción, la competición, la cooperación y el azar. }\end{array} \\ \text { EXPLICAR } & \begin{array}{l}\text { Relacionada con la clasificación, los argumentos, las formas de probar que algo ocurre, la } \\ \text { lógica, el relato y la conexión de las ideas. }\end{array}\end{array}$

Fuente: Oliveras y Gavarrete (2012, p. 362)

Tal como lo menciona Gavarrete (2012):

Además, a nivel global, tanto el trabajo de Gerdes (1996) como en el de Presmeg (2007), en el acontecer histórico de las actas de congresos internacionales (ICME e ICEm) y en los documentos respaldados por la UNESCO (1993, 1997 y 2012) se da continuidad a las siguientes ideas:

- Incorporar en el currículo elementos pertenecientes al entorno sociocultural, como punto de inicio para las propuestas didácticas escolares que propulsen el actor motivacional en la educación matemática. 
- Advertir a los profesores en formación inicial y en formación permanente, así como a los formadores de profesores, de la existencia de ideas etnomatemáticas en las prácticas sociales, que pueden aportar el desarrollo del currículo contextualizado activo que propone la visión funcional de las matemáticas, así como promover el respeto por la diversidad sociocultural.

- Incorporar en la formación profesional de profesores el componente de la investigación de ideas matemáticas de la propia cultura, etnia, comunidad lingüística y propiciar la incorporación de los hallazgos de estas investigaciones en los procesos de enseñanza.

- Incorporar en las propuestas curriculares material proveniente de varias culturas, de modo que se permita: valorar el contexto cultural de todos los estudiantes y profesores e incrementar su autonomía cultural, motivar el respeto por la visión multicultural y ampliar la comprensión de las matemáticas, así como valorar su contribución en las necesidades y actividades humanas.

\section{(...)}

- Elaborar material sobre el patrimonio etnomatemático e introducir los elementos culturales de las matemáticas en los libros de texto para facilitar el aprendizaje, respetar las diversidades y fortalecer las identidades. (p. 16)

Al respecto, Costa Rica es un país multicultural, en el cual se destacan diferentes grupos étnicos, como el de los indígenas autóctonos; sin embargo, existen otros grupos cuyas raíces se remontan a siglos atrás, como lo son la cultura limonense y la guanacasteca, que presentan características propias (comidas, vestimenta, idioma, arte, entre otras) muy diferentes a las del resto del país.

Además, en los últimos años, Costa Rica ha recibido una gran cantidad de inmigrantes provenientes de Venezuela, Colombia, Nicaragua y países asiáticos, de los dos últimos en números muy significativos, los cuales demandan respeto por sus raíces culturales basado en pautas de organismos internacionales como la UNESCO.

Lo anterior provoca, desde el punto de vista educativo, un problema que debe ser no solo analizado, sino planteado en propuestas curriculares concretas, ya que cada vez las situaciones escolares son más complejas y pueden llevar a roces sociales entre los diferentes grupos como sucede en otros países.

Si bien en Costa Rica el problema de la multiculturalidad está en la agenda de los partidos políticos y del MEP, la realidad es que poco se ha hecho en la práctica, pues entre los programas de estudio, por ejemplo, el de matemática no considera las características de las diferentes regiones del país y menos las de las minoría étnicas; asimismo, el calendario académico nacional, propuesto por el MEP, no toma en cuenta aspectos como: la religiosidad, cosmovisión y tradiciones de los pueblos indígenas.

Así, se evidencia la importancia de realizar propuestas curriculares concretas y pertinentes para atender la multiculturalidad y, en un futuro muy cercano, referirse a la sociedad costarricense como pluricultural.

\section{METODOLOGÍA}

El presente estudio corresponde a un diseño cualitativo de tipo etnográfico porque se involucra un grupo de personas que pertenecen a una misma cultura, por lo que sus relaciones presentan una base común a través del tiempo. Además, comparten costumbres, tradiciones, creencias; es decir, mantienen patrones de comportamientos. Así, en el artículo se describen algunos aspectos de ese estilo de vida que se relacionan con contenidos de la matemática.

A pesar de la diversidad de instrumentos para recolectar información, se escogió la entrevista semiestructurada, la observación y los grupos focales, lo cual responde a características fundamentales de 
la población en estudio, como son el dominio de la comunicación oral y las dificultades para la expresión escrita tanto en castellano como en su lengua natal. Los instrumentos aplicados se describen a continuación:

- Instrumento I. Guía de entrevista: Los participantes fueron maestros indígenas y no indígenas que trabajan en dos centros educativos: Villa Damaris y Alto Quetzal. Se aplicó con el fin de identificar aspectos relacionados con la cultura cabécar y la enseñanza de la matemática en primaria.

- Instrumento II. Guía de observación participante: Se aplicó con el propósito de anotar la información relacionada con elementos del entorno cabécar, por ejemplo con características de la infraestructura de la escuela y casas cercanas, actividades reproductivas y de la cotidianidad como son los procedimientos para cultivar granos y el diario vivir. La observación se efectuó durante un convivio organizado por estudiantes, maestros y padres y madres de familia de la Escuela Jaktain y sus alrededores.

- Instrumento III. Guía para grupo focal: Consistió en ocho preguntas abiertas, en las que los dos subgrupos explicarían, mediante dibujos y algunas frases, elementos o procedimientos cotidianos de la cultura cabécar que se relacionaran con las seis actividades matemáticas según Bishop, la técnica se aplicó en la Sede de la Universidad de Costa Rica en Turrialba.

La muestra fue intencional, ya que se requerían indígenas que conocieran la situación actual del sistema educativo de primaria en el área de la matemática; además, era necesario conocer dos ámbitos: centros a los cuales se llegara en carro y centros a los que el acceso solo existe caminando. Así, la muestra estuvo conformada por:

- Un maestro no indígena y un maestro de lengua y cultura cabécar de la Escuela Jaktain.

- Un maestro no indígena de la Escuela Villa Damaris.

- Tres maestros indígenas de la Escuela Alto Quetzal.

- Quince estudiantes de la carrera Bachillerato en Ciencias de la Educación en I y II ciclos con énfasis en Lengua y cultura cabécar (también maestros en ejercicio).

- Para la organización y codificación de la información se establecieron dos categorías, de las cuales a este artículo le competen dos:

- Nociones matemáticas en la cultura cabécar: Entendidas como ideas o concepciones de términos que impliquen medición, orden, tiempo, cantidad, tamaño, espacio, adición, sustracción, multiplicación y división.

- Elementos de la cosmovisión cabécar relacionados con contenidos matemáticos de l y ll ciclos de la EGB: Se refiere a elementos del entorno de los escolares cabécares como rituales, cotidianeidad, organización social, actividades productivas y reproductivas, relaciones interpersonales y relaciones con el medio. En ambas categorías se incluyen las seis actividades matemáticas universales señaladas por Bishop (1999): contar, localizar, medir, diseñar, explicar y jugar. Lo anterior a partir de los aspectos que se tratan en cada categoría.

\section{PRESENTACIÓN E INTERPRETACIÓN DE RESULTADOS}

Debido a que el estudio se refiere al pueblo cabécar es necesario mencionar algunos datos que permitan caracterizar a esta población. Según el Instituto Nacional de Estadística y Censo (2013), la etnia es la segunda en mayor número de habitantes, abarca ocho territorios a saber: Chirripó, Ujarrás, Tayni, 
Talamanca Cabécar, Telire, Bajo Chirripó, Nairi Awari y China Kicha, de los cuales en este artículo se trabajó solo con el primero. Chirripó cuenta con una población de 6341 habitantes, de los cuales se identifican como indígenas 5982, 93,3\% indican estar aseguradas, en su mayoría por el Estado. En cuanto a los niveles de escolaridad, $61,7 \%$ cuenta con primaria completa y tan solo un $15,6 \%$ con secundaria concluida. El empleo agrícola predomina en los hombres $(77,6 \%)$, las familias indican que $45,4 \%$ posee finca o parcela agropecuaria. Específicamente, las comunidades participantes en el estudio pertenecen a la provincia de Cartago y al cantón de Turrialba.

Seguidamente, se muestran los datos obtenidos en la investigación de acuerdo con las categorías enunciadas anteriormente.

\section{Nociones matemáticas en la cultura cabécar}

A continuación se mencionan algunas nociones matemáticas encontradas en la cultura cabécar que deben considerarse para el estudio de esta disciplina, ya que de alguna manera inciden en la forma cómo se propicia el aprendizaje de los contenidos obligatorios, en relación con recursos y estrategias didácticas por ejemplo.

Se observan nociones de plano en la construcción de la cocina, ya que, pues colocan tres piedras y encima los recipientes para preparar el alimento, lo que indica que para sostener un objeto o plano en el espacio se requieren tres puntos de soporte (tres puntos no alineados definen un plano o tres patas son suficientes para mantener una mesa en equilibrio).

Entre los elementos de la naturaleza y el entorno, existe una relación de respeto, de carácter divino, ya que hay rituales específicos para la obtención de favores.

No se tiene un horario estructurado de comidas ni de trabajo, e incluso no hay distinción entre un día entre semana o de fin de semana, tampoco existe un día específico para la adoración del Dios (Sibú). Cabe mencionar, que la distinción de los días de la semana lo impone el MEP, con la inserción de las escuelas en estas comunidades, lo que dificulta la puntualidad en la asistencia a clases. A lo anterior se refiere un maestro indígena:

"Los indígenas no se preocupan tanto por el tiempo, no tienen una hora específica para las comidas ni para el trabajo. No tienen un día específico de descanso, no discriminan entre los días de la semana para las labores productivas. También es común que durante la jornada lectiva los estudiantes vayan llegando a diferente hora porque los caminos no se mantienen igual todo el año." (Respuesta a Instrumento I)

El conocimiento y dominio de uno o varios contenidos matemáticos está implícito en el oficio que cada persona indígena práctica, y se aclara que en estas culturas no todas las personas tienen el don para ejercer ciertas profesiones, por ejemplo la de curandero, líder, sepulturero, entre otros. Sin embargo, el conocimiento se transmite en forma oral y de generación en generación según el don otorgado.

Los conocimientos matemáticos relacionados con algunas de las seis actividades señaladas por Bishop (1999) son:

\section{Contar}

Se evidencia en el diseño y construcción de la mochila, ya que su forma lo determina la cantidad de puntadas, cuatro o seis. El conteo, en forma empírica, es una de las actividades básicas de toda sociedad, 
pues se hacen cálculos aproximados de cuánto material se necesita para de la construcción de casas u otras estructuras. También se nota en la compra e intercambio de productos, ya sea entre miembros de la familia, o con otros pueblos, y según la edad, ya sea niño escolarizado o adulto no escolarizado.

El nombramiento y clasificación de objetos depende de la posición y presentación de estos, difiere si se encuentran solos o en grupos; en el caso de las aves se dicen de una manera si están volando (clase plana) y de otra si están en un árbol (clase redonda). Este aspecto dificulta los ejercicios como identificar cantidad de objetos en un grupo, realización de operaciones básicas, conteo, entre otros. El término "cuántos" también difiere si se refiere a objetos individuales o a racimos.

\section{Diseñar}

El diseño de las construcciones es de carácter mental y por experiencia (empírico y sin la utilización de instrumentos de medición precisos, desprovistos de escalas), puesto que no trazan previamente diagramas o esquemas.

\section{Jugar}

Durante los juegos se establecen diferentes relaciones, por ejemplo, el fútbol y las escondidas se realizan entre niños y niñas, pero no se involucran los adultos. En el "Escondido" se evidencia el dominio del espacio físico, ya que se dificulta encontrar a quienes se ocultan. Se amplía el tema según la clasificación de Bishop (1999):

- Realistas: Implica ejecutar una actividad real con objetos reales, en esta clasificación se ubicó "Tirarse al río desde las peñas", lo cual conlleva a aprender una estrategia.

- Imitativos: Consiste en imitar elementos de la naturaleza o acciones de los adultos. En este caso fue asumir roles de animales, producir los sonidos que emiten y ubicarse en la posición según corresponda, asíde cuatro patas en el caso del perro. Este tipo de juego contribuye al desarrollo de la creatividad y al razonamiento lógico.

- Discriminativos: "El escondido" se considera un juego muy común, consiste en buscar estrategias y desarrollar dominio del espacio para encontrar a los demás participantes.

- Propulsivos: Incluye movimiento y lanzamiento de objetos, ejercer una fuerza, en este grupo se ubicó "Deslizarse por las peñas" que implica el desarrollo de una estrategia para alcanzar el objetivo y calcular la fuerza de lanzamiento o movimiento.

- Imaginativos: Se mencionó la narración de historias, con la salvedad que es más común de los niños a los adultos; sin embargo se ven sometidos a mantener un rol de escucha específico.

- Disputativos: El fútbol, también conocido como "Jugar bola" implica esforzarse por quitar el balón a los miembros del equipo contrario y anotar goles. Implica el desarrollo de razonamiento lógico, entre otros aspectos.

\section{Elementos de la cosmovisión cabécar relacionados con contenidos matemáticos de I y II ciclos de la EGB}

En el primer ciclo, por ejemplo, en el área de Geometría (MEP, 2012, p.152) se propone desarrollar en los estudiantes la capacidad de visualizar las formas geométricas y algunas relaciones básicas entre ellas en el entorno en el que se presentan, así como iniciar un proceso de abstracción geométrica. 
Al respecto se exponen cada uno de los conceptos básicos obligatorios, las habilidades específicas por desarrollar y el aspecto de la cultura correspondiente según los resultados encontrados en este estudio.

TABLA 2

Conceptos básicos y habilidades específicas en líneas y segmentos

Grado

Primer año

Segundo año

Segundo año

Tercer año
Conocimientos

Conocimientos básicos:

Líneas rectas

Líneas curvas

$\begin{array}{ll}\begin{array}{l}\text { Líneas } \\ \text { Horizontal } \\ \text { Vertical }\end{array} & \begin{array}{l}\text { Identificar en dibujos y en el entorno posiciones de líneas } \\ \text { rectas: horizontal, vertical, oblicua }\end{array} \\ \begin{array}{ll}\text { Oblicua } \\ \text { Paralelos }\end{array} & \text { Trazar líneas rectas en posiciones horizontal, vertical, oblicua } \\ \text { Perpendiculares } & \begin{array}{l}\text { Reconocer segmentos y rectas paralelas y perpendiculares en } \\ \text { dibujos y objetos del entorno }\end{array} \\ & \text { Trazar segmentos paralelos y perpendiculares }\end{array}$

\section{Habilidades específicas}

Identificar y trazar líneas rectas y curvas

Fuente: Elaborado a partir de MEP (2012, pp. 110, 112 y 114).

Los tipos de línea se encuentran en el entorno indígena, en la forma de algunos animales como la culebra que representa una curva, también las rectas se visualizan en objetos de las edificaciones propias de las comunidades y en las tres posiciones (horizontal, vertical y oblicua). Además, dominan las nociones de perpendicularidad y paralelismo en forma empírica, lo que se evidencia al consultar cómo logran construir algunas edificaciones.

TABLA 3

Conceptos básicos y habilidades específicas en figuras planas, cuerpos sólidos y circunferencias

\begin{tabular}{|c|c|c|}
\hline Grado & Conceptos básicos & Habilidades específicas \\
\hline Segundo año & $\begin{array}{l}\text { Figuras planas } \\
\text { - } \quad \text { Triángulo } \\
\text { - } \text { Cuadrilátero } \\
\text { - } \text { Cuadrado } \\
\text { - } \text { Rectángulo } \\
\text { - Vértice } \\
\text { - Lado }\end{array}$ & $\begin{array}{l}\text { - Reconocer triángulos y cuadriláteros } \\
\text { - } \text { geométricos } \\
\text { - Reconocer si un cuadrilátero es un rectángulo } \\
\text { - Reconocer si un rectángulo es un cuadrado } \\
\text { - Identificar elementos de una figura plana (vértice, lado) } \\
\text { - Identificar semejanzas y diferencias en las figuras en triángulos, } \\
\text { cuadrados, rectángulos y cuadriláteros en general } \\
\text { - Componer y descomponer figuras utilizando cuadriláteros y } \\
\text { triángulos }\end{array}$ \\
\hline Segundo año & $\begin{array}{l}\text { Cuerpos sólidos } \\
\text { - Esferas }\end{array}$ & - Identificar objetos que tengan forma esférica \\
\hline Tercer grado & Circunferencias & - Identificar y trazar circunferencias \\
\hline
\end{tabular}

Fuente: Elaborado a partir de MEP (2012, pp. 112, 113, 115)

Las escuelas indígenas se construyen conservando la perpendicularidad y la forma tradicional rectangular en los pisos y paredes, las diferencia que se montan en basas por las condiciones del terreno (ver figura 1). De esta manera, no como parte de su tradición, pero sí de su entorno, se pueden estudiar algunas figuras planas (triángulos, rectángulos, entre otros). 


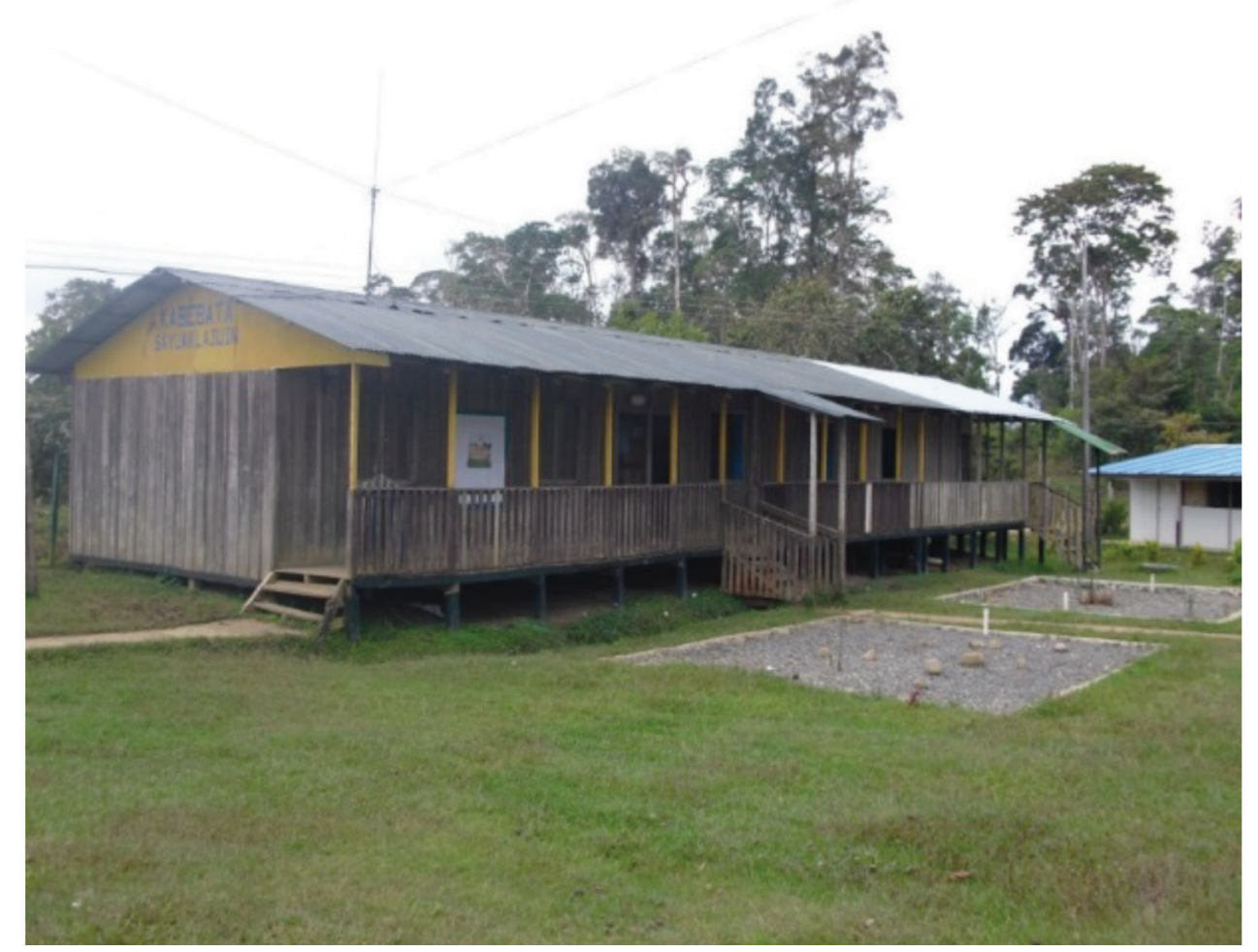

Figura 1. Estructura de una escuela en territorio indígena. Fuente: Archivo fotográfico de la autora.

Otro elemento con el cual se pueden estudiar figuras planas se encuentra en las artesanías como la canasta y los bolsos, pues las primeras se elaboran con base circular, rectangular, pentagonal o hexagonal y los segundos cuadrangulares o rectangulares; además de que el tejido también posee figuras planas. Otros elementos en los cuales se visualizan figuras y líneas son la cerbatana y la jícara.

Se debe tomar en cuenta que los indígenas no utilizan instrumentos de medición de tipo occidental, por lo que en primera instancia se recomienda el trazo de figuras de acuerdo con su forma natural de hacerlo, "se hace a través del cálculo visual, con observación y en forma empírica" (participante grupo focal).

A continuación se detallan los conocimientos encontrados con esta categoría y algunas de las actividades según Bishop (1999).

\section{Explicar}

Los ranchos tradicionales cabécar muestran, en su mayoría, la estructura en forma cónica, la cual por ser de este tipo posee base circular en el piso. Lo interesante es cómo la construyen sin un instrumento similar al compás. Según Sánchez y Velásquez $(2012$, p. 61) la argumentación obtenida es la siguiente (ver figura 2).

"Se coloca una estaca en cualquier punto de la superficie y con una cuerda atada, luego se gira alrededor formando 8 puntos con la misma distancia. Aquí la distancia de la cuerda la elige el constructor." (Participante grupo focal) 
"Se fija un poste central, tiran un bejuco de cierto tamaño y van marcando la posición de los postes en forma circular, de tal manera que los postes queden a la misma distancia del centro." (Participante grupo focal)

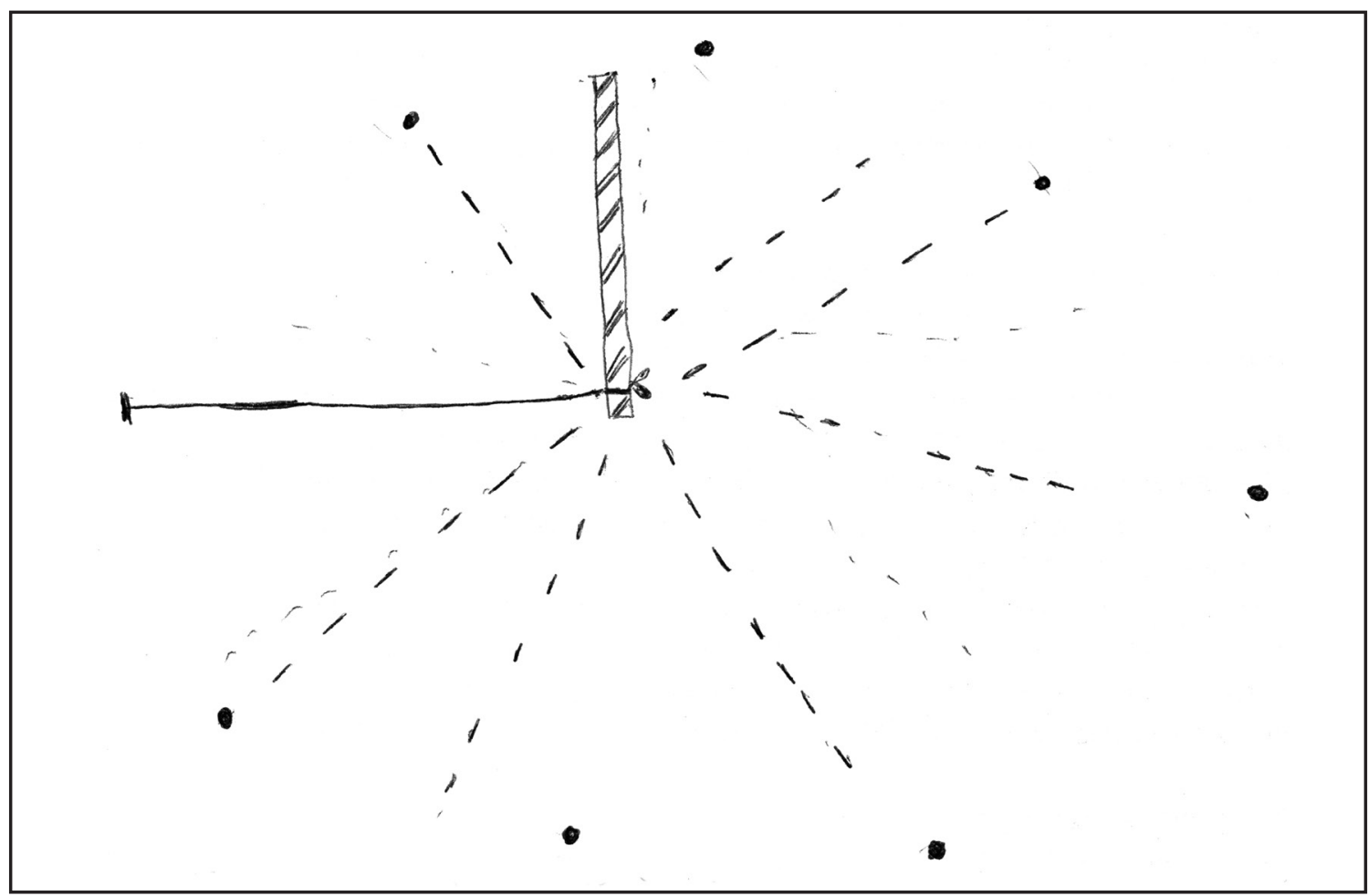

Figura 2. Construcción de un círculo. Fuente: Archivo fotográfico de la autora.

Cabe mencionar, que los ocho postes tienen una justificación mítica, pues durante la creación Sibö contó con la ayuda de ocho animales, al respecto Gavarrete (2012) indica que esto también se relaciona con los ocho sorbones que se cantan en el ritual de la purificación de la vivienda, al respecto González y González (2000, p. 68) mencionan que uno de sus informantes explicó la relación de la siguiente manera:

(...) el primero es el canto del perdicillo (suirere); el segundo es un Sorbón del zoncho (urú); el tercero es pavo; el cuarto chancho de monte; el quinto gavilán o águila que le llaman; el seis es tigre, el siete es un tipo de pájaro que le llamamos en Bribri sicú; el ocho es úca: seres que viven en las montañas grandes, son dos hombres pegados por la espalda, uno con la cara para un lado y el otro para otro. Se jalan, dicen, uno va allá y el otro para acá y así. Son ocho cantos que los cantores saben bien.

La forma cónica tiene una connotación religiosa, según los participantes se debe a dos aspectos:

"Es la misma forma del techo de la casa de Sibö." (Participante grupo focal)

"Esa forma representa el género masculino, así Sibö nos representó." (Participante grupo focal)

Tanto los cuerpos sólidos como las esferas se representan con el Sol y la forma similar de algunos frutos que se producen en los alrededores de la escuela, en el trayecto de la escuela a la casa y cerca del hogar. 
En el texto anterior, se nota la argumentación, lógica y conexión de ideas que utilizan los cabécares participantes al brindar el significado y el procedimiento para construir la base del rancho tradicional, demostrando así la actividad matemática universal "explicar".

\section{Localizar}

La tabla 4 incluye los datos del Programa de Estudios de Matemática oficial, que refiere al tema de "localizar".

TABLA 4

Conceptos básicos y habilidades específicas en posición-localización

\section{Grado Conceptos básicos \\ Habilidades específicas}

Tercer año

Posición- localización

Ubicar personas u objetos a partir de un punto de referencia

Fuente: Elaborado a partir de MEP (2012, p. 114).

En las figuras 3 y 4 se observan cuáles son los puntos de referencia utilizados comúnmente por los cabécares participantes, para indicar un trayecto de la escuela a su casa. Se identifican ríos, escuelas, último lugar donde llega el carro, duración en tiempo de una cuesta, entre otros.

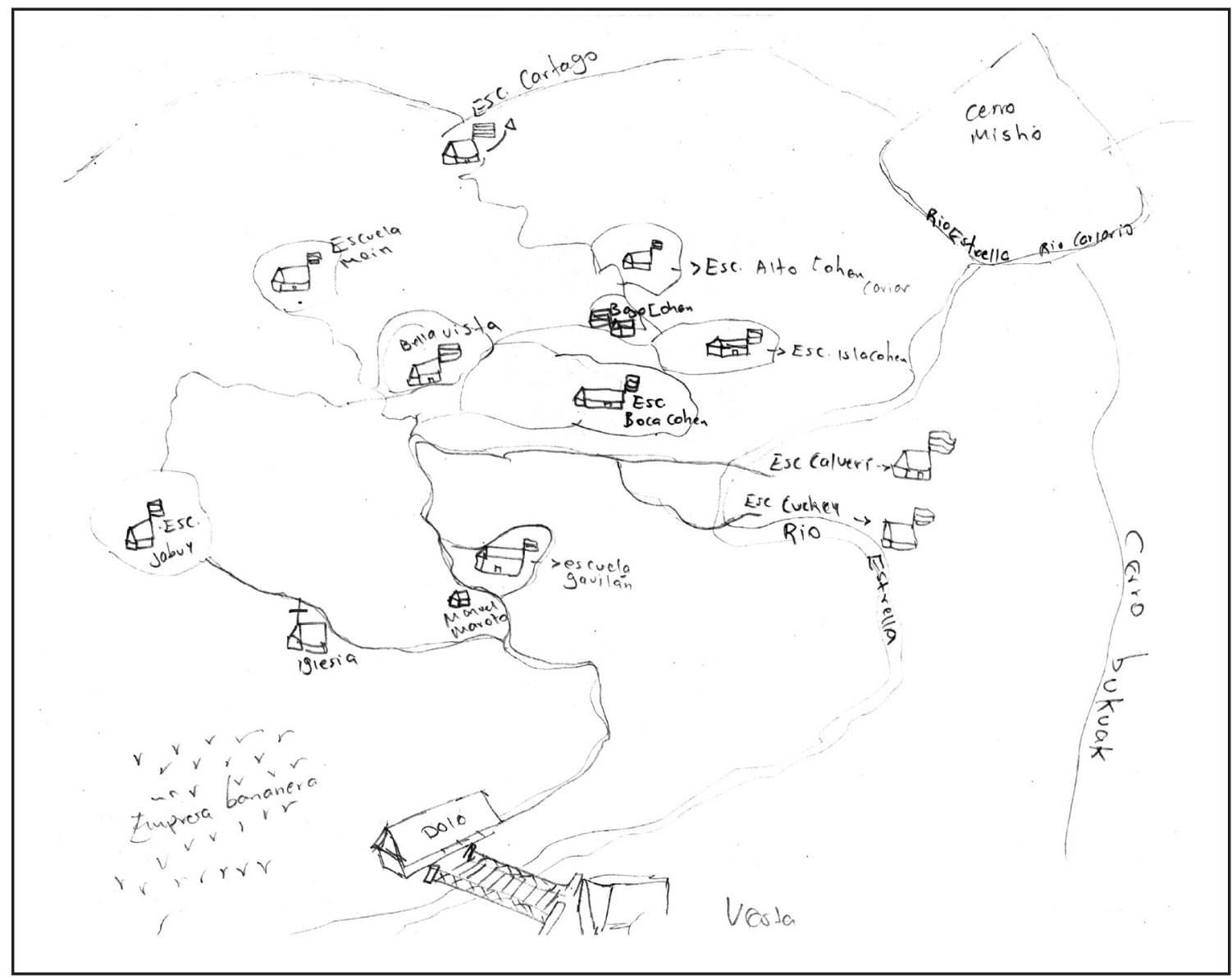

Figura 3. Croquis del Grupo 1. Fuente: Archivo fotográfico de la autora. 


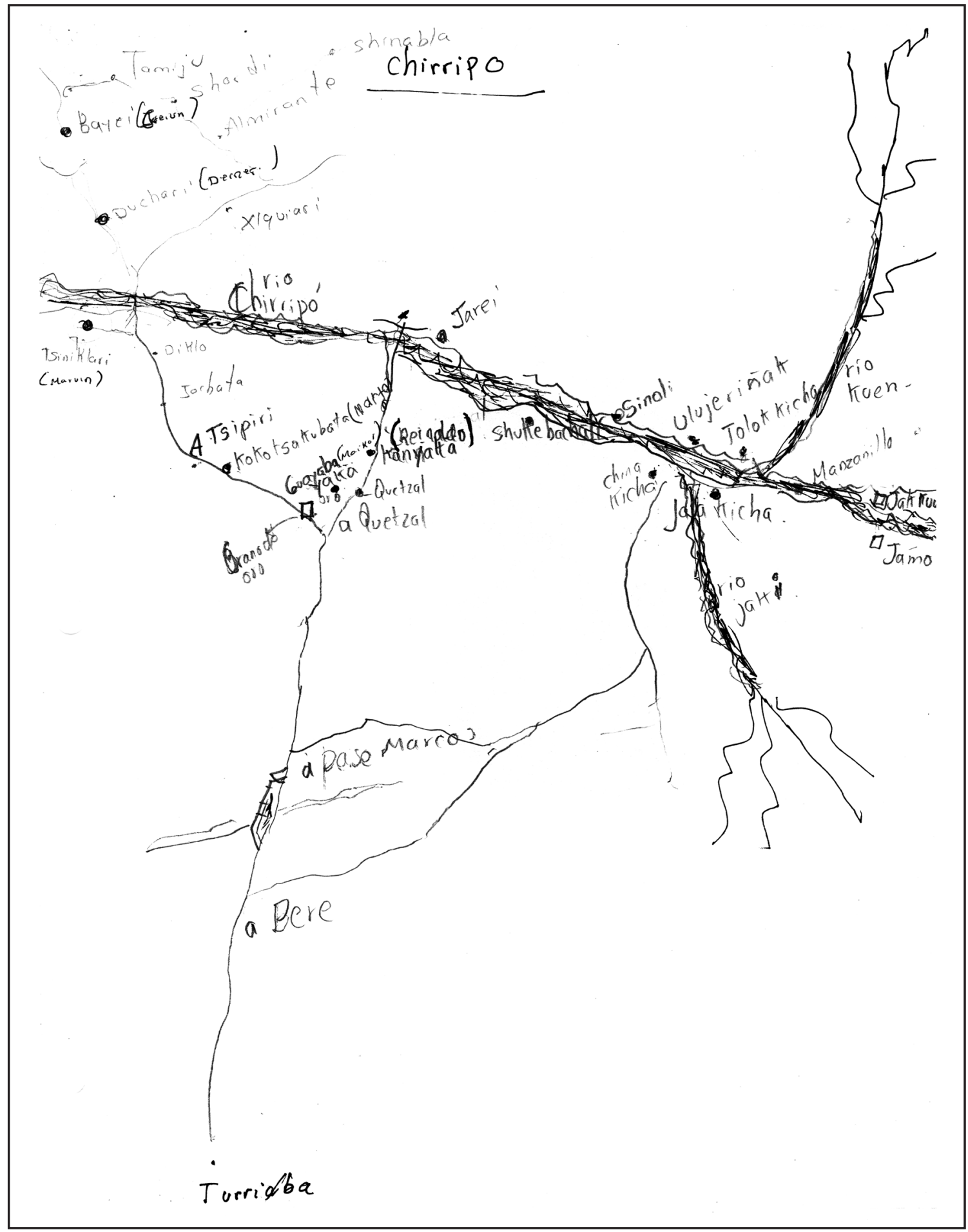

Figura 4. Croquis del Grupo 2. Fuente: Archivo fotográfico de la autora. 


\section{Medir}

Al referirnos al área de "medidas", en el primer ciclo el MEP (2012) propone que el estudiante inicie el proceso de comprensión del concepto de medida y que calcule, estime, compare y aplique algunas de ellas.

Se propone el estudio de varios conceptos y habilidades en cuanto a tamaño, noción de longitud anchura - espesor, moneda, peso, tiempo y capacidad, de los cuales la investigación base solo abordó ampliamente dos: distancia y tiempo, en menos profundidad: peso, volumen y moneda nacional.

La propuesta del MEP parte de unidades de medida como el metro y las horas y minutos para el tiempo, sin embargo, se debe tener presente que estás no son las comunes en las culturas indígenas, por lo que se menciona a continuación aspectos relevantes por considerar en el abordaje de ambos temas.

\section{Altura y tamaño}

En el caso de alguna construcción se utilizan como referencia partes o totalidad del cuerpo humano, por ejemplo, se dice: la altura de este rancho es dos veces "Juan", el tamaño de esta cuerda son tres brazos. Un ejemplo lo expresa un maestro indígena:

"... la tira para hacer las amarras se calcula con la mano entera, todas son del mismo tamaño, los mayores saben si se quiere para más de 4 años se le pone humo, el humo protege el mecate, lo seca y cuando cae lluvia no entra el agua." (Respuesta a Instrumento I).

\section{Distancia}

Si son muy cortas se usan pasos, varillas y otros materiales, si es larga como de la escuela a la casa, se relaciona con el tiempo que se dura en llegar caminando, es común oír: De aquí a allá hay dos horas y media.

\section{Tiempo}

No utilizan instrumentos como el reloj, sino que se guían por los sonidos que emiten algunos animales, como el canto de los gallos para levantarse y controlan el transcurrir del día por el canto de algunas aves, el anochecer llega con el sonido de las chicharras. Otro punto de referencia es la posición del sol y el número de estrellas para determinar la hora del día o la noche.

Es importante mencionar que los periodos de siembra se establecen de acuerdo con las fases de la Luna o las épocas de lluvia o sequía, por lo que no se refieren a un mes específico del año, así lo manifiesta un maestro indígena:

“Nosotros sembramos por la luna, si quiere sembrar yuca y malanga se siembra con la luna llena,... en luna menguante y creciente los animales andan por todo lado y se comen todo lo que se encuentran." (Respuesta a Instrumento I)

Los periodos como años, días, meses, hoy, mañana y pasado mañana se utilizan en la tradicional narración de historias que los mayores tienen con los miembros de la comunidad.

\section{Peso y Volumen}

No se logró ahondar más estos temas, pero se indica que para preparar las comidas, se determina la cantidad de cada ingrediente utilizando cucharas o "cálculos de ojo" (una pizca, un puñito); de igual forma 
en actividades agrícolas como la siembra de cultivos se menciona: el arroz por puños y los frijoles y el maíz por cantidad de granos.

\section{Moneda nacional}

Con la llegada de las pulperías a las zonas indígenas y la venta de productos agrícolas, los habitantes han tenido que familiarizarse con el uso de la moneda nacional, con lo cual se evidencia que principalmente los mayores no tienen interiorizadas las operaciones de adición y sustracción, ya que compran un producto y lo pagan, con el vuelto compran otro y lo pagan y así sucesivamente, hasta que se les acaba el dinero. A esto se refiere un maestro participante:

"Aquí hay pulperías, entonces ellos conocen el dinero, aquí ellos tienen más posibilidad de ver el dinero porque ellos manejan la platica más diferente que los de adentro,... siempre se hace una pulpería para empezar las sumas y todo eso las monedas, billetes y así no cosas tan complicadas." (Respuesta a Instrumento I).

Los ejemplos detallados anteriormente permiten demostrar que medir es común en las diferentes actividades propias de la cultura cabécar.

\section{Otros resultados}

A continuación se mencionan dos resultados relevantes, pero no relacionados estrechamente con las dos categorías planteadas.

Se evidencia preocupación por la pérdida de aspectos importantes de la cosmovisión cabécar (construcciones, artesanías, costumbres y tradiciones) con la penetración de la educación no indígena impartida por el MEP, además del aprendizaje de contenidos obligatorios no pertinentes en estos contextos, al respecto un docente manifiesta:

"Cómo hace uno para enseñar a los chiquitos qué son medios de comunicación si por estos lugares casi no hay, señales de tránsito que no existen aquí,... entonces el asunto es muy complejo." (Respuesta a Instrumento I)

Debido a las tradiciones de la cultura cabécar, en muchos casos las relaciones en el aula, e incluso la asistencia a las clases pueden ser afectadas. Por ejemplo, como la situación comentada por las madres cuando a sus niñas se les presenta el periodo (menstruación):

"Un día de estos vino una mamá y me dijo que no le dieran comida a su hija, que solo arroz podía comer y sin sal, que no podía compartir utensilios con otras personas y todo eso por tres días, otras no las dejan venir durante ese periodo ellos les llaman dietas." (Respuesta a instrumento I)

La situación descrita puede no ser entendida por el resto de compañeros y maestros no indígenas.

Un aspecto no menos importante que afecta cualquier proceso educativo si hay desconocimiento de las tradiciones culturales es la práctica de dietas, en las que la persona indígena debe estar aislada de sus semejantes o no puede practicar alguna actividad, esto ocurre en casos como: la llegada de la menstruación, el parto, el fallecimiento de un familiar, enfermedad, entre otros. 


\section{CONCLUSIONES Y RECOMENDACIONES}

Con base en el trabajo realizado se logró determinar una serie de elementos matemáticos en la cultura cabécar que se pueden incorporar en los planes de estudio de matemática a nivel de I y II Ciclo de la educación general básica costarricense, con el fin de contextualizarlos a la realidad de los pueblos indígenas, tal como la forma de contar y medir, por ejemplo.

También se establecen diferencias en concepciones de ciertos eventos por parte de poblaciones indígenas y no indígenas, porque las de las primeras son muy intuitivas, hecho importante de tomar en cuenta sobre todo en evaluaciones, pues los objetos animados, en muchos casos reciben diferentes nombres dependiendo de si están agrupados o en movimiento.

En síntesis, con los datos presentados es posible asegurar que las seis actividades matemáticas universales indicadas por Bishop (1999) son practicadas en la cultura cabécar de Chirripó y que tienen relación con contenidos estudiados en el sistema escolar costarricense.

De la investigación en general, se pudo determinar la necesidad de adecuar los programas de matemática, en este caso particular, a nivel de I y II ciclo de la EGB, que el Ministerio de Educación Pública desarrolla en las zonas indígenas, ya que los contenidos no mantienen estrecha relación con la cosmovisión de las zonas indígenas.

Por otro lado, se recomienda al Ministerio de Educación Pública aplicar otro tipo de estructura escolar (horarios, uniformes, efemérides, lengua, entre otras) consensuada con dichas poblaciones, pues la investigación evidencia el origen de la pérdida de aspectos tradicionales y culturales con la instauración en las zonas de centros educativos públicos que poseen la misma estructura aplicada en el resto del país.

La actual propuesta curricular en matemática, a nivel de primaria y secundaria plantea la resolución de problemas como estrategia metodológica central y la contextualización como estrategia didáctica, pero no contempla la multiculturalidad, por lo que se recomienda plantear la formación de docentes, ya sea provenientes de los grupos minoritarios o de otras zonas dispuestos a entender, conocer y convivir con estos grupos, en especial, con los pueblos indígenas.

\section{REFERENCIAS}

Bishop, A. (1999). Enculturación matemática, la educación matemática desde una perspectiva cultural. Barcelona: Paidós.

Bishop, A. (2000). Enseñanza de las matemáticas: ¿cómo beneficiar a todos los alumnos? En N. Gorgorió, A. Deulofeu y A. Bishop (Coords), Matemáticas y educación: Retos y cambios desde una perspectiva internacional, pp. 35-56. Barcelona: Graó.

Bozzolli, M. y Guevara, M. (2002). Los indígenas costarricenses en el siglo XXI. San José: EUNED.

D'Ambrosio, U. (2008). Etnomatemática. Eslabón entre las tradiciones y la modernidad. México: Limusa.

Naciones Unidas (2008). Declaración de las Naciones Unidas sobre los derechos de los pueblos indígenas. Recuperado de http://www.un.org/esa/socdev/unpfii/documents/DRIPS_es.pdf

González, A. y González, F. (2000). La casa cósmica talamanqueña y sus simbolismos. San José: EUNED.

Ministerio de Educación Pública. (2012). Programas de estudio de Matemática I y ll ciclo de la Educación Primaria, III ciclo de la Educación General Básica y Educación Diversificada. Recuperado de: http:// www.mep.go.cr/sites/default/files/programadeestudio/programas/matematica.pdf 
Gavarrete, M. (2012). Modelo de aplicación de Etnomatemáticas en la formación de profesores para contextos indígenas en Costa Rica. Recuperado de http://www.etnomatematica.org/publica/trabajos_ doctorado/tesis_gavarrete.pdf

Gavarrete, M. (2006). Etnomatemática en el territorio Talamanca Bribri. Ponencia presentada en el V Festival Internacional de Matemática. Recuperado de http://www.cientec.or.cr/matematica/pdf/RGavarrete.pdf

Instituto Nacional de Estadística y Censos. (2013).X Censo Nacional de Población y VI de Vivienda:Territorios indígenas. Recuperado de: http://www.uned.ac.cr/extension/images/ifcmdl/02._Censo_2011._ Territorios_Indigenas.pdf

Olivé, L. (2004). Interculturalismo y justicia social. México: UNAM.

Oliveras, M. y Gavarrete. M. (2012). Modelo de aplicación de Etnomatematicas en la formación de profesores para contextos indígenas en Costa Rica. Revista Latinoamericana de investigación en Matemática Educativa, 15 (3), 339-372.

Sánchez, A. y Velásquez, K. (2012). Educación para todos: La cultura cabécar y la matemática en I y ll ciclos de la Enseñanza General Básica en Costa Rica. Informe de investigación.

Schmelkes, S. (2006). La interculturalidad en la educación básica. Ponencia presentada en la Segunda Reunión del Comité Intergubernamental del Proyecto Regional de Educación para América Latina y el Caribe (PRELAC), Santiago de Chile, 11 al 13 de mayo de 2006. Recuperado de http://www.socolpe.org/data/normalarmenia/BIBLIOGRAFIA/interculturalidad_educacion_basica_schmelkes.pdf 